\title{
WLAN-WIMAX SCHEDULER-BASED HYBRID SENSOR NETWORK FOR DATA AGGREGATION IN SMART GRID
}

\author{
Rajashree T. Daryapurkar ${ }^{*}$ and R. G. Karandikar ${ }^{2}$ \\ ${ }^{1}$ Electronics \& Telecommunication Engineering, Thadomal Shahani Engineering \\ College, Bandra, K. J. Somaiya College of Engineering, Vidyavihar, Mumbai, \\ Mumbai University, India \\ ${ }^{2}$ Electronics \& Telecommunication Engineering, K. J. Somaiya College of \\ Engineering, Vidyavihar, Mumbai, Mumbai University, India \\ ${ }^{1}$ rajashreedaryapurkar@somaiya.edu, ${ }^{2}$ rameshkarandikar@ somaiya.edu
}

\begin{abstract}
Smart meters, sensors and other measuring devices in smart grid networks are expected to generate huge amounts of data continuously. Data generated from these devices must be delivered reliably to utility control centers within specified time for wide-area monitoring and control. Overall grid status would be determined by using $t$ his data in a timely manner. Hence reliable and efficient data aggregation is an important process in smart grid communication network. Several network designs have been proposed for data aggregation using various communication technologies. WiMAX and WLAN are the two promising technologies of wireless networks. The combined operation of WiMAX and WLAN can extend transmission range and significantly improve the efficiency of a hybrid network by improving link quality. Hybrid network allows flexible data aggregation to reduce signaling and protocol overheads. We implemented multilevel WLAN WiMAX communication environment for data aggregation in smart grid to support multiple traffic sources/ applications using Network Simulator3 and analyzed the performance of the network in terms of quality of service parameters specified for smart grid applications. Results indicate that proposed network architecture can satisfy the delay requirements of multiple smart grid traffic sources/ applications.
\end{abstract}

Keywords - WLAN, WiMAX, Hybrid Sensor Network, Data aggregation, Smart grid, Network Simulator3

\section{INTRODUCTION}

Communication network is an essential component of smart grid. Data generated by sensors, smart meters and other devices needs to be collected and utilized by various smart grid applications. Data aggregation can be done using either wired or wireless communication technologies. Wireless communication is a main component to connect the digital world by overcoming the set of restriction factors from other communications. Wireless networks give technological advantages especially easy deployment. One of the key motives for choosing a wireless network is improving the communication concreteness of countryside subscribers that holdup after the city subscribers. WLAN technology based on the IEEE 802.11 standard is considered for communication in smart grid due to its low cost and wide use. [1] To make sure the longer connectivity up to several miles using IEEE 802.11 based network, we need a static wireless transportation technique based on IEEE

Received: May 7, 2019

Reviewed: August 19, 2019

Accepted: August 28, 2019

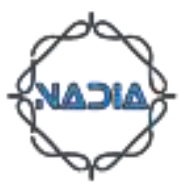


802.16 known as WiMAX. Features like security and scheduler support makes it applicable for the smart grid communication. [2, 3] Hybrid networks can provide better QOS and are preferable for advanced metering application of smart grid. [4, 5] Hybrid network with WiMAX can provide scalable, cost effective solution to satisfy the QOS requirements of various applications of smart grid. [6,7] Wireless sensor networks are important part of smart grid infrastructure used for control and monitoring functions. Optimizing every form of WSN communication and networking is the overall objective. [8, 9] To provide QOS requirements of different smart grid application along with the delay critical applications is the goal of network design for smart grid communication. [10]

There are several methods to understand the output of big wireless communications using measurements from the network to get the most correct results. Sometimes we need to consider the scalability, the multiple scenarios of placements, various packet rates at nodes, dissimilar position of devices. An optional method to know the output is using open source network simulators. Network Simulator 3 is open source software to carry out research in the field of networking. The simulation resolves the issue of changing the multiple environments to be calculated very efficiently. Multiple simulation situations can be built and data can be measured by the tool to assess the output of the networks with complex environment where output statistics have to be collected for different parameters. $[11,12]$ In this paper, we implemented scheduler based multilevel data aggregation hybrid sensor network using network simulator 3. We analyzed the performance of multilevel WLAN -WIMAX based network which can support multiple applications/traffic sources in smart grid. The rest of this paper is prearranged as follows. In Section 2, presented available existing work with WLAN-WIMAX standards for smart grid. In Section 3, the network design method that have been implemented using the Network Simulator-3 environment for Smart grid application is explained. In Section 4, results are presented and discussed. At the end, conclusions are presented in Section 5.

\section{EXISTING WORK WITH WLAN-WIMAX STANDARDS FOR SMART GRID}

Advanced metering infrastructure, substation automation, distribution automation, demand response management, distributed energy resources, remote site communication, and wide area situational awareness are some of the applications in smart grid. Advanced metering infrastructure or smart metering is used to transfer data from a smart meter to regional aggregator or meter collection point. Advanced metering infrastructure (AMI) uses real-time two-way communications between the utility and the customers. This system ensure optimal usage of energy, enable customer participation and enhance overall reliability, security and efficiency of the power grid A robust communication network is required for a successful AMI formation [7]. Various wired as well as wireless communication technologies are proposed for the designs of the network for data aggregation. Having fiber-optic backbone network extending it to thousands of homes can be one of option for utilities, but it will be costly for solely utility purposes. In contrast, the present-day wireless technologies are mature enough to provide reliable data communication to a large number of users. Moreover, they are fast and relatively easy to install and do not require major construction effort or structural interference in buildings. WiMAX technology has various advantages like seamless communications, high data rates scalability, lower cost of deployment, well developed security protocols, and long network span. The wireless local area networks (WLAN) have been widely used for short range communications, mainly based on the IEEE 802.11 (Wi-Fi) standard. [13, 14]Wireless LAN can be considered for various smart grid applications, such as control of distributed energy resources, distribution automation, Protection, and monitoring of a substation especially for remotely located small substation and Distributed Energy Resources. WiMAX and WLAN are potential wireless technologies to build AMI networks. [15, 16] 
Direct and aggregated architecture models with WIMAX technology for multiple services in smart grid are analyzed in urban, suburban and rural environment. Aggregation model make a better reliability between different traffic classes and collision probability can be reduced the for UDP traffics. As all the devices need not be activated by WIMAX technology, aggregation models are more economic. [17] WiMAX based traffic priority model for smart grid connecting Neighborhood Area Network (NAN) and Home Area Network (HAN) serving five different applications with parameters namely overall network delay, throughput and network capacity are studied. [18] The optimized configuration of Wi-Grid profile to meet the time critical smart grid communication requirements for the choice of frame duration, type-of service to traffic mapping, scheduling strategies, as well as the system architecture is investigated [19] QOS parameters for different applications like the electric car metering and pricing application, video surveillance and lastly voice support are analyzed.[20] One of the options is IEEE 802.11s-based wireless mesh networks to implement Neighborhood Area Networks (NANs) in Smart Grid (SG).[21] Specific results for IEEE 802.11af performance for neighborhood area network in a Smart Grid scenario are obtained.[22]

In an AMI network there would be a large number of measurement devices in order to collect readings from each residential and commercial meter within its coverage area. A low data connection may be needed in most of the Advanced Meter Reading applications but very high signaling and protocol overhead is required per transmission. Loss of signal strength at variable propagation conditions such as obstructions in the harsh utility environment, multipath, fading, background noise etc. limits the coverage area of a wireless network under the AMI environment In addition, some of the meters operating with fixed wireless links may always remain under poor coverage area To improve coverage of the network, a possible solution could be a denser distribution of base stations (BS) or access points (AP). However, it may further increase interferences and degrade the propagation environment. Low cost relays in the network, such as the IEEE 802.16j based WiMAX multichip relays could be another solution. But the relays complicates the network infrastructure and also increase interference, transmission latency and signaling overhead. Hybrid network architectures can provide strict quality of service (QoS) requirements of electric system automation applications in a cost-effective manner and are alternative for scalable wireless communication systems. Heterogeneous deployment of long-range IEEE 802.16 based WiMAX and short-range wireless technologies such as the IEEE 802.11 based WLAN networks can significantly enhance the performance and coverage of an AMI network [23].

Automation and management of distribution network has an important role to improve efficiency, reliability and quality of electric grid. Distribution network in smart grid can have types of devices like smart meters, sensors, voltage regulators, switches, transformers etc. In addition to collection of data from smart meters, sensors, distribution system has devices control, protection, configuration and monitoring functions. Controlling the power devices remotely, protection of part of distribution system after the fault occurrence and reconfiguration of devices remotely are control, protection and reconfiguration functions. Monitoring is one of function in distribution network used to transfer information from a feeder meter aggregation point to a sub-station. This is needed to monitor the status of feeder in terms of voltage, current etc. for operational and maintenance purposes. These functions give rise to control, protection, configuration and monitoring type of traffics. These traffics have different communication requirements. To carry traffic for these applications, WIMAX-WLAN based network can be utilized Wide area situational awareness is used for monitoring of power-system components and performance across the network over large geographic areas in near real time. The objectives of situational awareness are to understand and optimize the management of power components, prevent, or respond to problems before interruptions can occur. To provide communications to under serviced utility premises permanent and temporary sites, such as remote offices, 
substations, and construction sites is the goal of remote site communication application. Smart meters, sensors, voltage regulators, switches, transformers are considered to be the nodes in the network [24].

The dynamic spectrum access in heterogeneous wireless networks is a candidate technology that can be used to reduce the communication expenses (by reusing the existing infrastructures) and to improve overall performance of smart grid. [25] Over the broadband unified network, QoS can be provided for real-time and non-real-time traffic. [26] Userlevel QoS support can be provided with IEEE 802.11e WLANs. The multi-hop networks of IEEE 802.16 can provide an excellent network architecture for QoS support to different services. [27] Due to the advantages with the hybrid network, requirements of smart grid applications and importance of data aggregation technique, we considered multilevel data aggregation using hybrid network.

\section{MULTILEVEL DATA AGgREGATION USING HYBRID NETWORK}

The network is designed in three parts as network connectivity, application selection and scheduling and data aggregation. Network connectivity describes how a set of users are connected to the utility center. WiMAX supports different scheduling policies. Depending on the type of application, a scheduling policy is selected. Finally data aggregation algorithm used to collect the data through multiple levels is explained.

\subsection{NETWORK CONNECTIVITY}

The set-up environment as shown in Figure 1, is assembled using a hierarchical structural design connected to a set of users. Four level of hierarchical architecture based WiMAX-WLAN communication is formed in order.

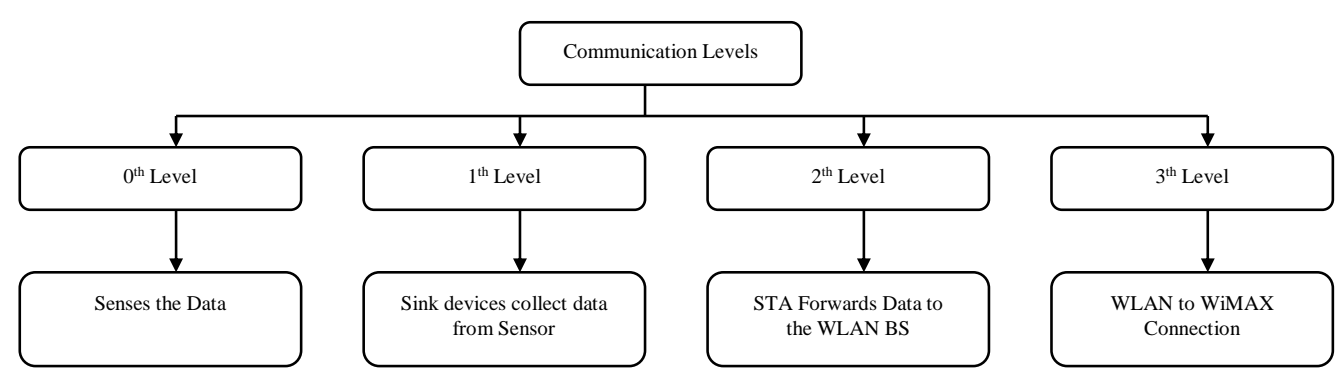

Fig. 1 Network Connection

In the 0th level, sensors senses the data from the environment such as electrical current $E_{C}$, voltage changes reading $V_{R}$, current, temperature $C_{T}$ state and pressure level $P_{E}$ for the operational and maintenance purposes from its deployed environment. 


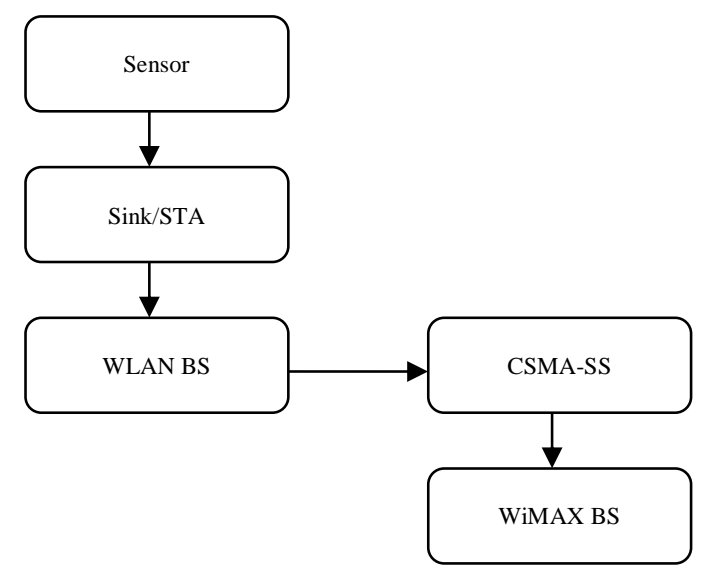

Fig. 2 Network Connectivity

In the first level, the sink devices are deployed and station devices STA maintains the connection with the sensor and observe the data from the network region. In the second level the STA forwards the data to the WLAN base station BS as shown in Figure. 2 WLAN nodes, the set of users served up by the WLAN BS is aggregated at a contact point as in Figure.3. The BS works as a gateway device for the set of users. Each 802.11 network with the BS along with the relevant users are attached to a cell. Each end point of the network allows the connectivity to one or more 802.11 cells. These each cell may be extending over the coverage of some kilometer distances. The WLAN BS of the network is connected to the CSMA node is connected with the 802.16 WIMAX BS. At the final level the WLAN BS maintains the communication with the WIMAX BS through the above network communication.

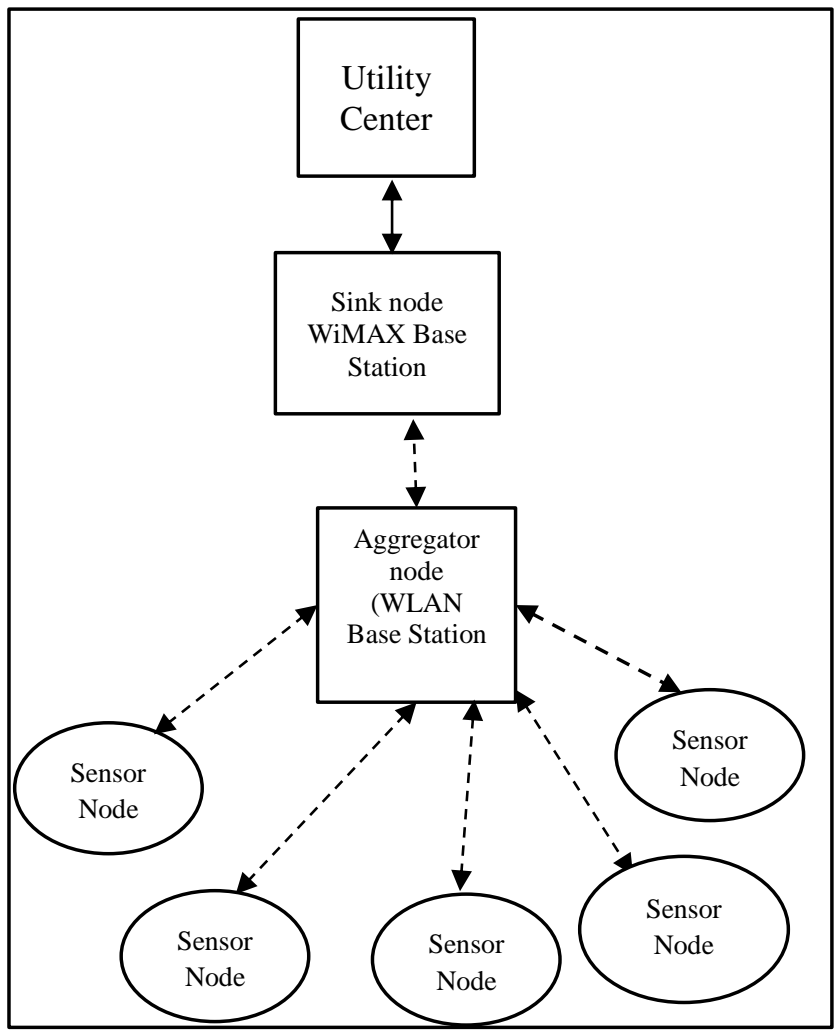

Fig. 3 Aggregation Model. 


\subsection{SCHEDULER MANAGEMENTS}

We recognized the behaviors and needs related with each data and plan a scheduling policy based on the time and consistent incoming significant data. The formulated scheduler also assures that the data is reasonably collected from preset nodes within the similar data class and is effectively collected by the destination within the convinced deadline. Packet frame period approximately assumed from 2.5 to 20 mille-second is divided into a downlink sub-frame and an uplink sub-frame and total packet frame period is depends on the kind of orthogonal frequency division multiplexing (OFDM) physical layer.In this design, the lowest level sensors are responsible to collect the data from the smart meters and subsequently send them to the service station schedulers based on the allocated service classes. All midway nodes worked as an aggregators and pass on to the downlink and uplink, in order. Five types are service classes are assigned to service station schedulers depend on the sensor applications.

The WLAN base station nodes acts as a terminal station which is used to collect the data from the sensors based on the sensed application and executes the data forwarding process. The following data observation and transmission knowledge is built inside the sensors during deployment period. The base model of the smart grid sensing process is developed with the smart metering application which collects and sent the data to the terminal station. The monitoring application sensors are collects the information related to the current, voltage temperature and pressure for the operational purpose. Smart meters $S_{M}$ are placed in smart grids to manage the energy sources, to progress the reliability. Figure 4 shows the functional section of a $S_{M}$ sensing and transferred to the WLAN base station, to create local communication. Accurate electric power sensing at the link between $S_{M}$ and the received mains electrical energy supply keeps the key to computing electric consumption data.

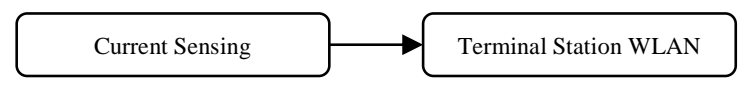

Fig. 4 Communication between Nodes

The remote monitoring and control application sensor fetches the information about the field-automated devices used for the distribution automation. The protection sensors execute the coordination application which fetches the location information by using the nearby broadcasting. The remote configuration and the access for the field devices are managed by the configuration sensor. The defense sensors permits the maintenance state of the network by reconfiguring the components in the shared network after complicated situations, so that the consistency and elasticity is to be enhanced by means of defense sensor monitoring. WiMAX end user equipment are built with the recloser, which is necessary for high voltage rated circuit_breakers with integrated current and voltage sensors and a defense relay, optimized for network shared defense asset. Improved defense management allows the error regions to infer with perfect accuracy, reduces the volume of end users without electric power, and gives advanced public protection. Isolated configuration setup is screening or adjusts product settings for damage assessment.

Based on the application type, the TCP/UDP connection is selected along with the service classes. The pre-emptive priority service class scheduling is applied with respect to the service class of the application. To validate the data connection type for the specific applications with their needed consistency, transmission control protocol TCP connections are used. By nature TCP connections are considered as high priority connectivity. 
However, in some cases the data that needs very low delay with high data rate; user datagram protocol UDP transmission is used for this type of classes as low priority transmission.

\title{
3.3. INTER-INTRA CLASS SERVICES
}

The high precedence and low precedence packets can arrive simultaneously so that a pre-planned guidelines is employed by the devices in order that by the arrival of a high precedence packets, the transmission of a low precedence ends up instantly. Once the transmission of low precedence data completion, then the pre-planned guideline is employed in the inter class service. In the intra class service, round robin and the fair queue model is employed to complete the data transmission. During the packet arrival, bytes per frame are computed from the minimum reserved traffic rate with the product of the frame duration per bit. As the incoming packet classes, especially control and monitoring, are important for the stability of the grid, so that we use a precedence based forecast policy in both WLAN base station and WiMAX base station. The precedence based scheduler believes the forecasting type precedences across all the devices as shown in Figure 7.

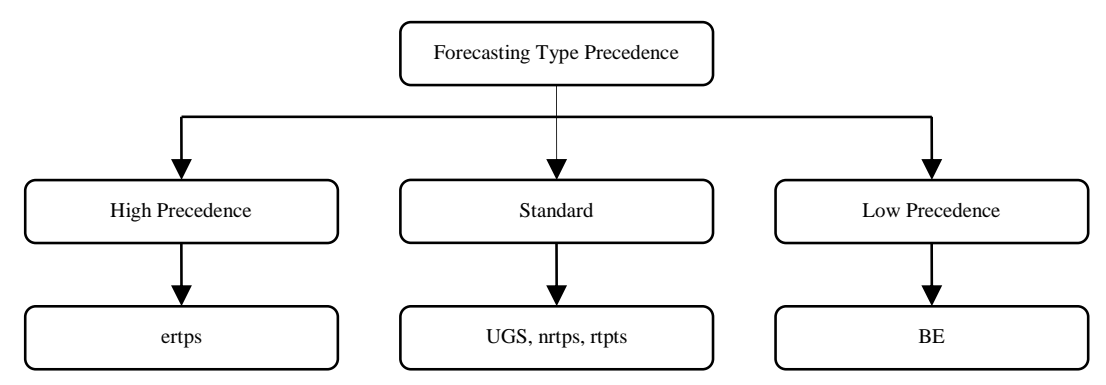

Fig. 7 Service Levels in Network

Since control and monitoring are both arrived unwanted contribution and they need low delay, so that we assigned an earliest deadline forecast guidelines in advance. While real time Polling Service control packet go after an arbitrary distribution and it also needs low delay, we assigned a round robin mode and hand out it as per the subjective fair queuing forecasting guideline so that no data streams are served at the same time, and we provide an advanced influence to the service streams with earliest deadline having higher data rate. Hence assign static bandwidth to the low precedence data and in round robin mode only if earliest deadline is still obtainable after allocating the high precedence streams.

\subsection{DATA AGGREGATION}

In this aggregation part, sensors are liable to collect the data from the smart meter and then send this data to the WIMAX base station through the WLAN base station. So the midway devices work as aggregators and carrier to downlink and uplink in order. Consequently, there would be some impacts can occur during the aggregation state compared to the direct delivery based on the scalability. Following are the steps for data aggregation.

\author{
Data Storage \\ Store Data [] \\ Update Data Count \\ Count++; \\ $\Sigma$ Data [i]; \\ Find Average ()
}




$$
\begin{aligned}
& \text { Average }=\frac{\sum \text { Data }[]}{\text { Count }} \\
& \text { Aggregated Data }=\text { Average }
\end{aligned}
$$

As above steps initially store data and update counts of the data by incrementing the values. Then sum the total data and update the sum values. By finding the average value using sum divided by count get the final aggregated values for each data.

Another fundamental side is that the power consumption of the smart meter observed by the sensors and transmit that data to the WIMAX base station is minimized by the aggregators, thus reduced the congestions and redundancies by compressing the data due to the relationship between the collected packets.

The data is aggregated using the multi-level aggregation using each level of hierarchy. The first level data aggregation is performed in the sensor to sink communication at which the sink collects and aggregate the data based on the sensing event type. The data collected from smart meter is directed to the aggregator, which exists at the same range of local transformer which is used to connect smart meter to the power grid. The second level aggregation is executed in the WLAN level with the collected data. The end level aggregation is performed by the WiMAX base station from the entire set of collected data. Upon receiving the data in each service class, the inter class and intra class is performed by based on the selected scheduling policy.

Table I. Total Number of Aggregation Packets

\begin{tabular}{|c|c|c|c|c|}
\hline \multicolumn{5}{|c|}{ Total Aggregation } \\
\hline Node & rtps Aggregation & nrtps Aggregation & BE Aggregation & UGS Aggregation \\
\hline 20 & 190 & 242 & 75 & 7 \\
\hline 25 & 272 & 134 & 145 & 62 \\
\hline 30 & 236 & 181 & 181 & 43 \\
\hline 35 & 248 & 282 & 148 & 8 \\
\hline 40 & 337 & 254 & 103 & 66 \\
\hline
\end{tabular}

Table II. Number of TCP aggregation packets

\begin{tabular}{|c|c|c|c|c|}
\hline \multicolumn{5}{|c|}{ TCP Aggregation } \\
\hline Node & rtps Aggregation & nrtps Aggregation & BE Aggregation & UGS Aggregation \\
\hline 20 & 156 & 215 & 69 & 3 \\
\hline 25 & 125 & 71 & 50 & 32 \\
\hline 30 & 171 & 138 & 124 & 35 \\
\hline 35 & 165 & 161 & 121 & 8 \\
\hline 40 & 139 & 116 & 56 & 17 \\
\hline
\end{tabular}

Table III. Number of UDP aggregation packets

\begin{tabular}{|c|c|c|c|c|}
\hline \multicolumn{5}{|c|}{ UDP Aggregation } \\
\hline Node & rtps Aggregation & nrtps Aggregation & BE Aggregation & UGS Aggregation \\
\hline 20 & 34 & 27 & 6 & 4 \\
\hline 25 & 147 & 63 & 95 & 30 \\
\hline 30 & 65 & 43 & 57 & 8 \\
\hline 35 & 83 & 121 & 27 & 0 \\
\hline 40 & 198 & 138 & 47 & 49 \\
\hline
\end{tabular}




\section{RESULTS AND DISCUSSION}

We have presented the simulation results and graphs for the implemented network. We performed simulations to determine the QOS provided by the network in terms of packet delivery ratio and average delay, considering different parameters of the network. Figure 8 and 9 shows the effect of variation of packet interval on packet delivery ratio and average delay as various applications in smart grid can have variation in packet interval value. We considered packet delivery ratio as it is measure of reliability of the network and average delay is important from the point of view of smart grid applications.

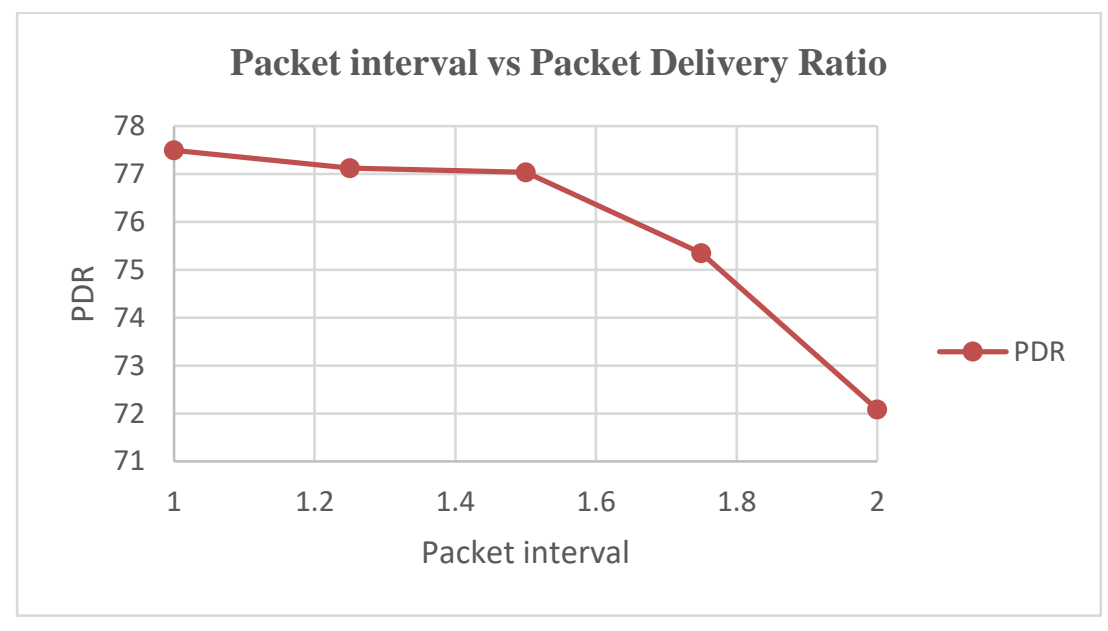

Fig. 8 Packet interval vs Packet Delivery Ratio

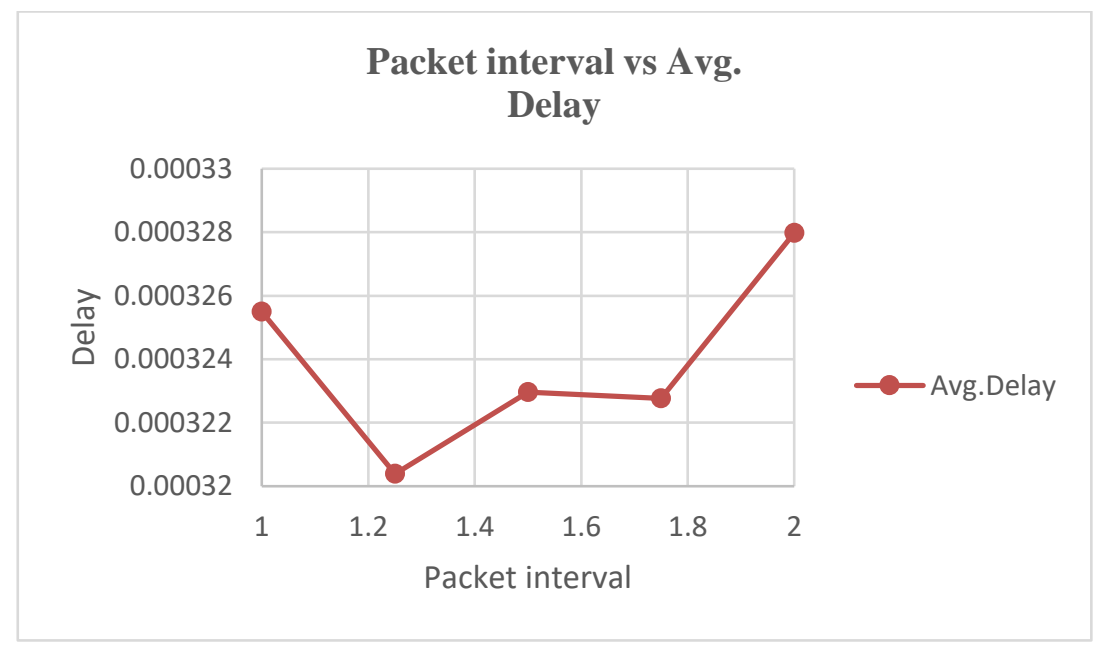

Fig. 9 Packet interval vs Average Delay

Figure 10 and 11 shows packet delivery ratio and average delay with respect to number of nodes in the network 


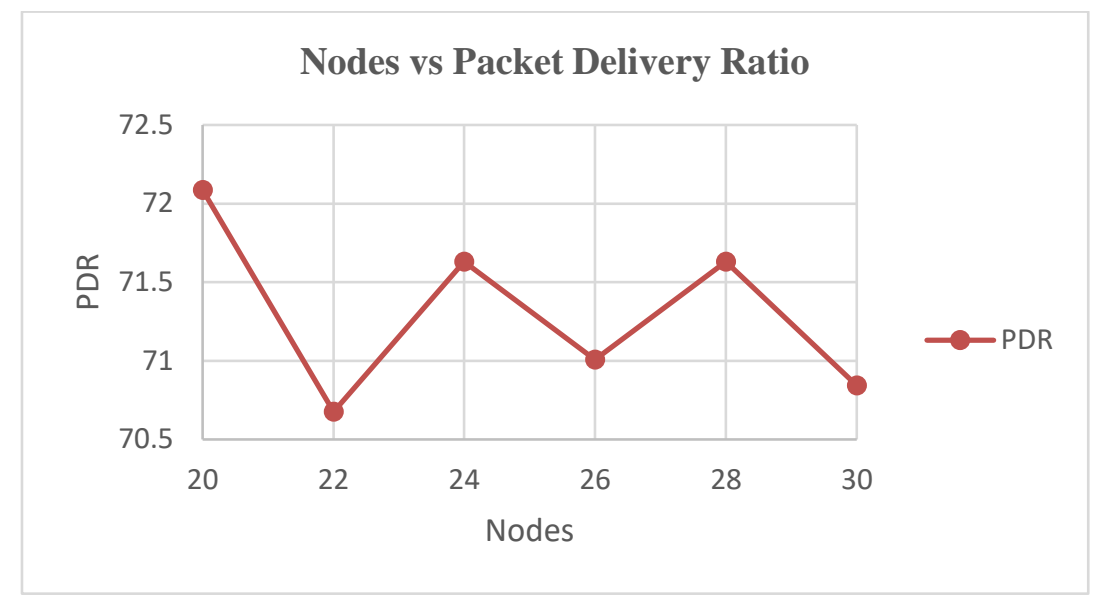

Fig. 10 Nodes vs Packet Delivery Ratio

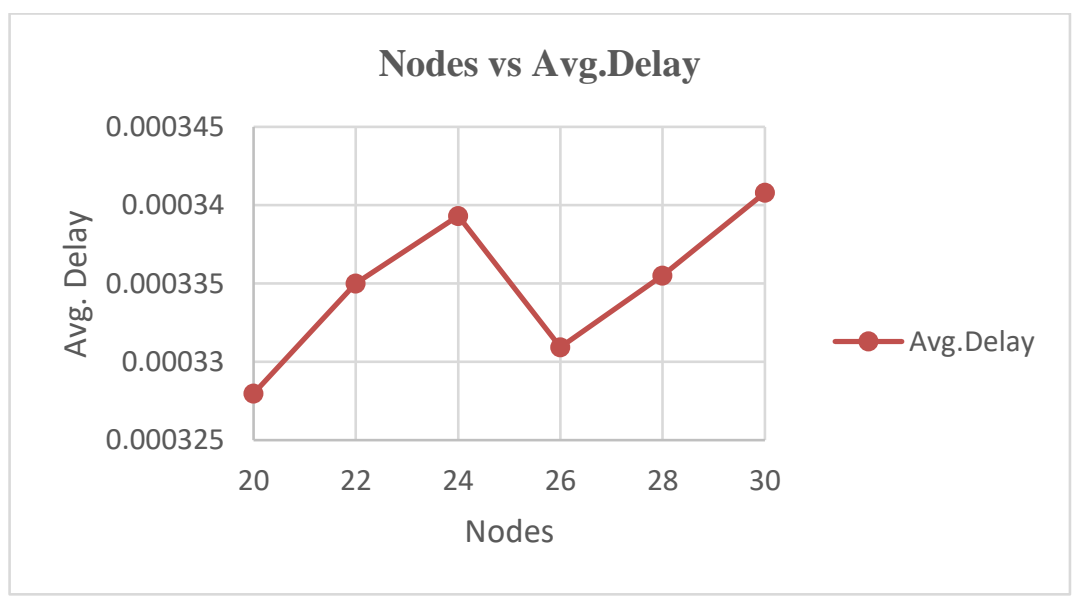

Fig. 11 Nodes vs Average Delay

Figure 12 and 13 shows packet delivery ratio and average delay with respect to simulation time.

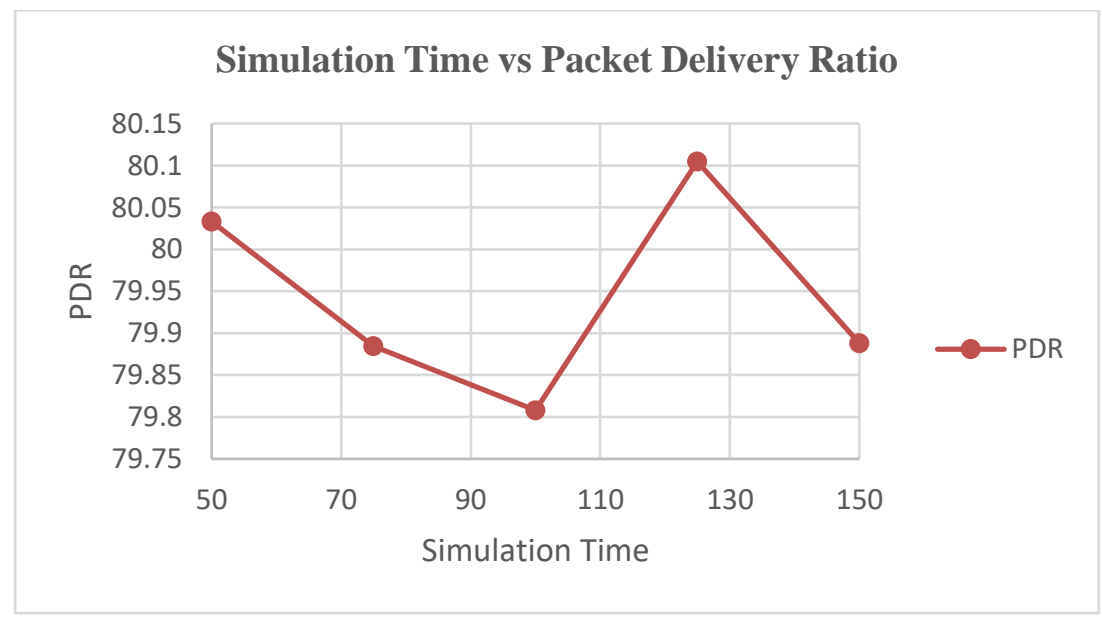

Fig. 12 Simulation Time vs Packet Delivery Ratio 


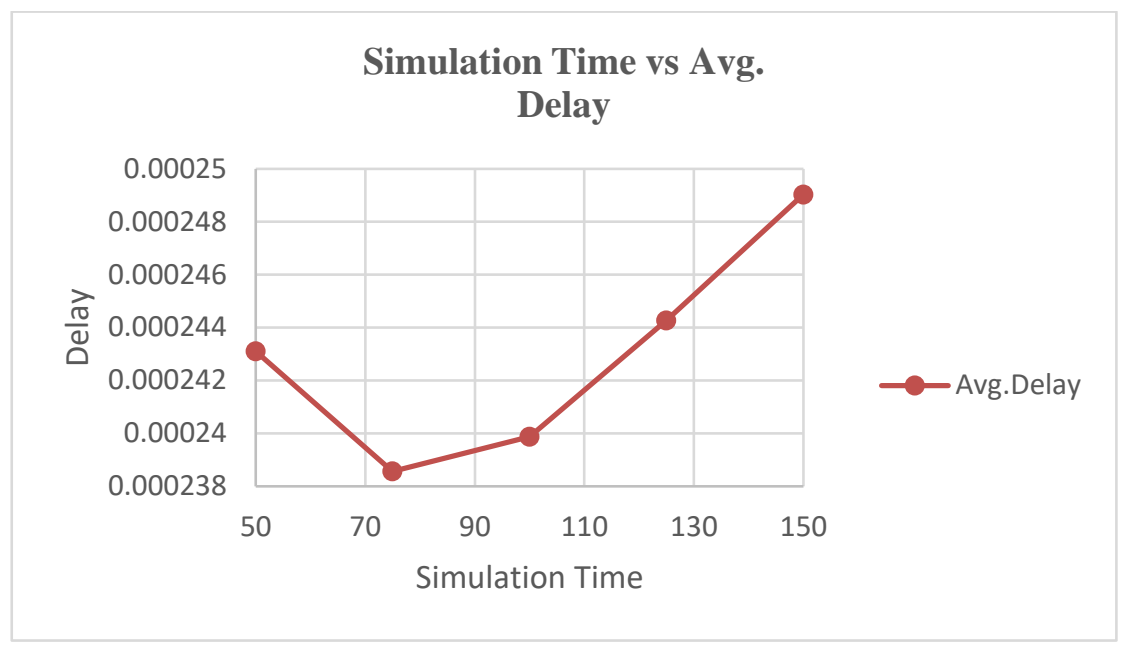

Fig. 13 Simulation Time vs Average Delay

Figure 14 and 15 shows packet delivery ratio and average delay with respect to variation in packet sizes. Different packet sizes can be used to support different applications in smart grid.

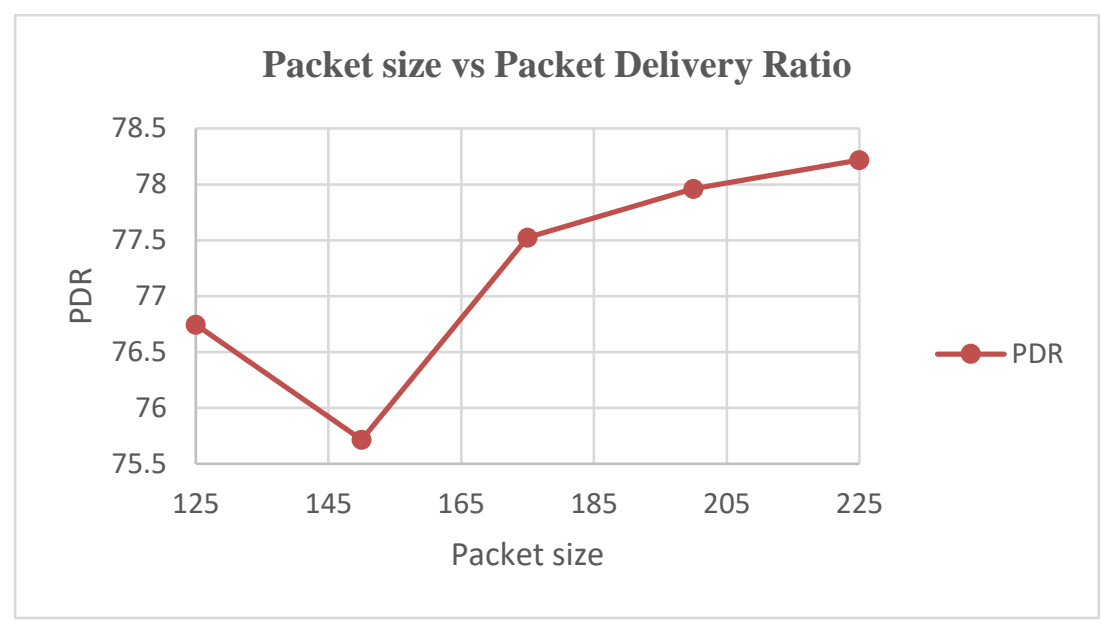

Fig. 14 Packet size vs Packet Delivery Ratio

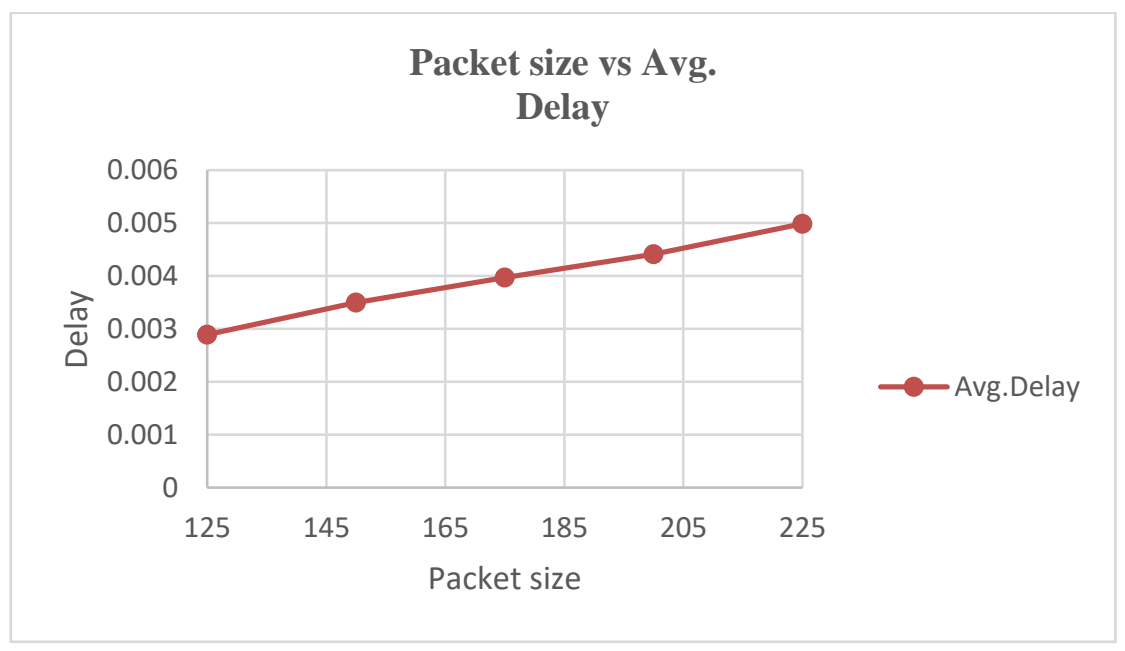

Fig. 15 Packet size vs Average Delay 


\section{CONCLUSION}

Gathering data from different sensors and devices within specified time is needed for proper functioning of smart grid. In the existing work, performance using technologies WLAN, WiMAX and WLAN-WiMAX hybrid networks are analyzed. Either local or single level aggregation is utilized to implement the network considering any one of the smart grid application. Traffic types and their QOS requirements for typical smart grid applications are specified in the existing literature. We implemented multilevel WLAN-WIMAX scheduler based hybrid sensor network for data aggregation in network simulator 3.It can support multiple traffic sources/ applications. Type of scheduling is chosen depending on traffic type /application. As WiMAX is utilized, range of network increases. We analyzed QOS performance of the network under normal condition. From the results, it can be observed that average delay values are less as compared to the delay values for similar network architectures discussed in existing work. Further work can be to analyze QOS performance of the network for fault and recovery conditions of the sensor network.

\section{REFERENCES}

[1] D. Baimel and S. Tapuchi, N. Baimel, "Smart Grid Communication Technologies- Overview,Research Challenges and Opportunities," International Symposium on Power Electronics,Electrical Drives, Automation and Motion, June 2016, Pages: 116 - 120, https://ieeexplore.ieee.org/document/7526014

[2] Veeranna Daravath, Aruna Daravath, "WI MAX (IEEE 802.16) Broad Band Technology For Smart Grid Applications, "International Conference on Communications and Signal Processing (ICCSP), April 2015, Pages: 1273 - 1275, https://ieeexplore.ieee.org/document/7322712

[3] Ban A. Al-Omar, Taha Landolsi, and A. R. Al-Ali, "Evaluation of WiMAX Technology in Smart Grid Communications," Journal of Communication, Vol. 10, No. 10, October 2015, https://pdfs.semanticscholar.org/ca8a/5c9a4f958807cb70cd2ad586ac4d119b292f.pdf

[4] Agustin Zaballos, Alex Vallejo, and Josep M. Selga, University Ramon Llull, "Heterogeneous Communication Architecture for the Smart Grid, "IEEE Network, Volume: 25, September/October 2011, Pages: 30 - 37, https://ieeexplore.ieee.org/document/6033033

[5] Esteban Inga, Sandra Céspedes, Roberto Hincapié, and Cesar Andy Cárdenas, "Scalable Route Map for Advanced Metering Infrastructure Based on Optimal Routing of Wireless Heterogeneous Networks," IEEE Wireless Communications, Volume: 24, Issue: 2, April 2017, Pages: 26 - 33, https://ieeexplore.ieee.org/document/7909154

[6] Thameemul Ansari. H, PremKumar. S, Saminadan. V, "Heterogeneous Network Modeling for Smart Grid Technology," International Conference on Communication and Signal Processing, April 6-8, 2016, Pages: 2336 - 2339, https://ieeexplore.ieee.org/document/7754118

[7] V.C. Gungor, F.C. Lambert, "A Survey On Communication Networks For Electric System Automation," The International Journal of Computer and Telecommunications Networking, Volume 50, Issue 7, 15 May 2006, Pages 877-897 dl.acm.org/citation.cfm?id=1648631

[8] Miao Li, Xiao-Bo Chi, Xin-Chun Jia, Jun-Li Zhang, "WSN-Based Efficient Monitoring for Overhead Transmission Line in Smart Grid," Proceedings of the 35th Chinese Control Conference, July 27-29, 2016, Pages: 8485 - 8489, https://ieeexplore.ieee.org/document/7554711

[9] Sinan Kurt, Huseyin Ugur Yildiz, Melike Yigit, Bulent Tavli, and Vehbi Cagri Gungor, "Packet Size Optimization in Wireless Sensor Networks for Smart Grid Applications," IEEE Transactions On Industrial Electronics, Vol. 64, No. 3, March 2017, Pages: 2392 - 2401, https://ieeexplore.ieee.org/document/7605474

[10] Irfan Al-Anbagi, Melike Erol-Kantarci and Hussein T. Mouftah, "Delay Critical Smart Grid Applications and Adaptive QoS Provisioning," IEEE Access, Volume 3, 2015, Pages: 1367 - 1378 , https://ieeexplore.ieee.org/document/7182266

[11] Ahmed. A. A Das, Naif. D. Alotaibi, Ahmed. N. Alotaibi, "Simulation of Wide Area WiMAX Networks in Urban Areas," International Journal of Advanced Computational Engineering and Networking, Volume-5, Issue-11, Nov.-2017, http://www.iraj.in/journal/journal_file/journal_pdf/3-415151628015193-97.pdf

[12] Adisorn Kheaksong, Akara Prayote and Wilaiporn Lee, "Performance Evaluation of Smart Grid Communications via Network Simulation version 3," 13th International Conference on Electrical Engineering/Electronics, Computer, Telecommunications and Information Technology (ECTI-CON) July 2016, Pages: 1 - 5, https://ieeexplore.ieee.org/document/7561360

[13] Afshan Mulla, Jaypal Baviskar, Smith Khare, Faruk Kazi, "The Wireless Technologies for Smart Grid Communication: A Review," 2015 Fifth International Conference on Communication Systems and 
Network Technologies, June 2015, Pages: $442-447$ IEEE Xplore ieeexplore.ieee.org/xpl/topAccessedArticles.jsp?punumber=727733

[14] Amar Patel, Juan Aparicio, Nazif Tas, Michael Loiacono and Justinian Rosca, "Assessing Communications Technology Options for Smart Grid Applications," IEEE International Conference on Smart Grid Communications, Oct. 2011, Pages. 126 -131 ieeexplore.iee.org/document/6102303

[15] V. Cagri Gungor, Dilan Sahin, Taskin Kocak, Salih Ergut, "A Survey on Smart Grid Potential Applications and Communication Requirements, "IEEE Transactions On Industrial Informatics, Vol. 9, No. 1, February 2013, Pages: 28 - 42 ieeexplore.ieee.org/document/6298960

[16] Palak P. Parikh, Mitalkumar. G. Kanabar, Tarlochan, S. Sidhu, "Opportunities and Challenges of Wireless Communication Technologies for Smart Grid Applications," IEEE PES General Meeting, July 2010, Pages: 1 - 7 ieeexplore.ieee.org/document/5589988

[17] Fariba Aalamifar, and Lutz Lampe, "WiMAX Technology in Smart Distribution Networks: Architecture, Modeling, and Applications," IEEE PES T\&D Conference and Exposition, April 2014, Pages: 1 - 5 ieeexplore.ieee.org/document/6863432

[18] P.Ponni shanmuga Priya and V.Saminadan, "Performance Analysis of WiMAX based Smart Grid Communication Traffic Priority Model ,'International Conference on Communication and Signal Processing, April 2014, Pages: 778 - 782 ieeexplore.ieee.org/document/6949949

[19] Fariba Aalamifar and Lutz Lampe, "Optimized WiMAX Profile Configuration for Smart Grid communications," IEEE Transactions on Smart Grid, Volume: PP, Issue: 99, March 2016, Pages 1-10 ieeexplore.ieee.org/document/7433415

[20] ana Neagu and Walaa Hamouda, "Performance of WiMAX for Smart Grid Applications," International Conference on Selected Topics in Mobile \& Wireless Networking (MoWNeT),April 2016, Pages 1-5 ieeexplore.ieee.org/document/6949949

[21] Nico Saputro and Kemal Akkaya, "Periodic Data Reporting Strategies for IEEE 802.11s-based Smart Grid AMI Networks," IEEE International Conference on Smart Grid Communications, Nov 2014, Pages: 314 - 319 ieeexplore.iee.org/document/7007665

[22] Huamiao Hu, Dritan Kaleshi, Angela Doufexi, Li Li, "Performance Analysis of IEEE 802.11af Standard based Neighbourhood Area Network for Smart Grid Applications," IEEE 81st Vehicular Technology Conference ,May 2015, Pages: 1 - 5 ieeexplore.ieee.org/document/7146000

[23] Reduan H. Khan, Jamil Y. Khan, "A Heterogeneous WiMAX-WLAN Network for AMI Communications in the Smart grid," IEEE Third International Conference on Smart Grid Communications, Nov 2012, Pages: 710 - 715 ieeexplore.ieee.org/document/6486070

[24] Rajashree Daryapurkar, R. G. Karandikar, "WiMAX for data aggregation in smart grid communication network-A review," International Conference on Wireless Communications, Signal Processing and Networking, pp. 97-100, 2017 ieeexplore.ieee.org/document/8299727

[25] Chandra Bajracharya and Danda B. Rawat, "Opportunistic Spectrum Access Enabled Heterogeneous Wireless Networking for Smart Grid," 13th IEEE Annual Consumer Communications \& Networking Conference (CCNC), Jan 2016, Pages: 793 - 797 ieeexplore.ieee.org/document/7444882

[26] G.Arul Prasath, K.R. Raghu, Maode Ma, "Integration of WLAN and WiMAX with Base Station Assisted QoS," 5th IFIP International Conference on Wireless and Optical Communications Networks, May 2008, Pages: 1 - 5 ieeexplore.ieee.org/document/4542483

[27] Myounghwan Lee and John A. Copeland, "An Adaptive Polling Algorithm with Differentiation Strategy in IEEE 802.16 Multi-hop Networks with IEEE 802.11e WLANs," IEEE 28th International Performance Computing and Communications Conference, Dec 2009 Pages: $354-359$ ieexplore.ieee.org/document/5403850 
International Journal of Future Generation Communication and Networking Vol. 12, No. 3 (2019) 